\title{
Transcranial magnetic stimulation and
} transcranial direct current stimulation appear to be safe neuromodulatory techniques useful in the treatment of anxiety disorders and other neuropsychiatric disorders

\author{
Uso clínico da estimulação magnética transcraniana e da estimulação transcraniana por \\ corrente direta em transtornos de ansiedade e de transtornos neuropsiquiátricos \\ Aline lannone', Antonio Pedro de Mello Cruz¹, Joaquim Pereira Brasil-Neto², Raphael Boechat-Barros ${ }^{3}$
}

\begin{abstract}
Transcranial magnetic stimulation (TMS) has recently been investigated as a possible adjuvant treatment for many neuropsychiatric disorders, and has already been approved for the treatment of drug-resistant depression in the United States and in Brazil, among other countries. Although its use in other neuropsychiatric disorders is still largely experimental, many physicians have been using it as an off-label add-on therapy for various disorders. More recently, another technique, transcranial direct current stimulation (tDCS), has also become available as a much cheaper and portable alternative to TMS, although its mechanisms of action are different from those of TMS. The use of off-label therapeutic TMS or tDCS tends to occur in the setting of diseases that are notoriously resistant to other treatment modalities. Here we discuss the case of anxiety disorders, namely panic and post-traumatic stress disorders, highlighting the uncertainties and potential problems and benefits of the clinical use of these neuromodulatory techniques at the current stage of knowledge.
\end{abstract}

Keywords: transcranial direct current stimulation; anxiety; panic disorder.

\section{RESUMO}

A estimulação magnética transcraniana (TMS) foi recentemente proposta como um possível tratamento adjuvante para muitos distúrbios neuropsiquiátricos, e já foi aprovada para o tratamento de depressão fármaco-resistente nos Estados Unidos e no Brasil, entre outros países. Apesar do fato de que seu uso em outros transtornos neuropsiquiátricos ainda é em grande parte experimental, muitos médicos têm utilizado essas técnicas como uma terapia off-label em várias doenças. Mais recentemente, uma outra técnica, a estimulação transcraniana por corrente contínua (ETCC), tornou-se também disponível como uma alternativa muito mais barata e portátil do que a TMS, embora os seus mecanismos de ação sejam diferentes daqueles da TMS. O uso off-label de TMS ou ETCC tende a ocorrer no caso de doenças que são notoriamente resistentes a outras modalidades terapêuticas. Aqui nós discutimos o caso dos transtornos de ansiedade, ou seja, transtorno do pânico e estresse pós-traumático, destacando as incertezas, benefícios e problemas potenciais inerentes ao uso clínico dessas técnicas neuromoduladoras no atual estágio do conhecimento.

Palavras-chave: estimulação transcraniana por corrente contínua; ansiedade; transtorno de pânico.

Transcranial magnetic stimulation (TMS) and transcranial direct current stimulation (tDCS) are noninvasive transcranial stimulation techniques recently used in the fields of neurology and psychiatry. Originally introduced by Barker, Jalinous and Freeston ${ }^{1}$, TMS has consolidated itself as an increasingly important technique for noninvasive stimulation of the human motor cortex ${ }^{2}$. Initially used as a method to investigate changes in motor pathways due to neurological diseases and even certain neuropsychiatric disorders ${ }^{3}$, TMS also began to be used as a form of therapy for diseases such as

'Universidade de Brasília, Instituto de Psicologia, Brasília DF, Brasil;

${ }^{2}$ Universidade de Brasília, Instituto de Biologia, Departamento de Ciências Fisiológicas, Laboratório de Neurociências e Comportamento, Brasília DF, Brasil; ${ }^{3}$ Universidade de Brasília, Faculdade de Medicina, Departamento de Psiquiatria, Brasília DF, Brasil.

Correspondence: Joaquim P. Brasil-Neto, Departamento de Ciências Fisiológicas da Universidade de Brasília; Campus Darcy Ribeiro; $70910-900$ Brasília DF, Brasil; E-mail: jbrasil@unb.br

Conflict of interest: There is no conflict of interest to declare.

Received 30 March 2016; Accepted 21 June 2016. 
Parkinson's ${ }^{4}$, and epilepsy ${ }^{5}$. In the last two decades, research on using these tools to treat conditions such as depression ${ }^{6}$, mania ${ }^{7}$, obsessive-compulsive disorder ${ }^{8}$ and post-traumatic stress disorder ${ }^{9}$ began to appear.

The use of electric currents for treating psychiatric disorders began in the $18^{\text {th }}$ century with the development of the voltaic pile. However, it was only in the 1960s and 1970s that the noninvasive method of cerebral stimulation known as brain polarization, similar to modern tDCS, was able to provide improvements in mood and alertness in healthy volunteers, in addition to treating depression ${ }^{10}$. Later, this method was abandoned, possibly due to the advance of psychopharmacology and the social stigma of electroconvulsive therapy that hindered the development and emergence of new noninvasive forms of cerebral stimulation.

Despite the controversies involving such an early noninvasive method of cerebral stimulation, tDCS began to be used again as a putative neuromodulatory tool in the present century, notably with the studies of Priori ${ }^{10}$ in Italy, and Nitsche and Paulus ${ }^{11}$ in Germany. Both studies were able to demonstrate that the transcranial induction of a low-intensity direct current, through electrodes placed on the head, increased (anodal stimulation) or decreased (cathodal stimulation) cortical excitability during the period of stimulation, suggesting that tDCS probably produced its effects through changes in the resting membrane potential ${ }^{12}$, thus modifying the threshold for firing of action potentials, or even through synaptic mechanisms.

As a neuromodulatory technique, its physical and physiological principles require less complex equipment than TMS, with only two electrodes being necessary: one cathode and one anode, which, arranged in different positions, create a flow of low-intensity ( 1 or $2 \mathrm{~mA}$ ) direct electric current that covers a specific region of the cerebral cortex, modulating it in accordance to the polarity. The electric current, in turn, flows from one electrode to the other through the scalp and the cortex. By comparison, while TMS can generate strong currents capable of depolarizing the neuron until it reaches the threshold for firing action potentials, tDCS changes cortical activity through weak electric currents, producing changes in the resting membrane potential, and consequently in brain activity ${ }^{13}$.

One fact that stands out in tDCS is the duration of its physiological effects. The technique is able to decrease or increase cortical excitability for hours after stimulation ${ }^{11}$, probably by inducing long-term depression or long-term potentiation on the treated neurons and synapses ${ }^{14}$. Due to these long-term changes in cortical excitability, it is commonly applied daily, for 20 to 30 minutes $^{15}$, ensuring efficacy and safety. Taking into account its prolonged effects upon cortical excitability, low intensity of the currents used to modulate brain activity, as well as the fact that it also allows sham stimulation in experimental protocols ${ }^{16}$, tDCS has been widely accepted not only as an off-label treatment but also as a research tool, instead of high-cost equipment such as magnetic stimulators. Furthermore, the technique appears to be quite safe, and there is no reason to-date to suspect tDCS to be detrimental to health ${ }^{17}$, since it presents a low rate of side effects when used in accordance with the standard procedures recommended by recent clinical studies for the treatment of psychiatric disorders. The only side effects associated with tDCS have been redness of the skin or mild superficial electrolytic burns ${ }^{18}$. However, due to the still short follow-up time of treated subjects, longer-term side effects, if any, are unknown at this time.

\section{APPLICATIONS OF TMS AND TDCS IN PSYCHIATRY}

While in the past it was necessary to surgically manipulate the brain in order to modulate its activity in a nonpharmacological way, noninvasive stimulation provides something new: through TMS and tDCS, it is possible to adjust cerebral activity and apparently even mental processes, with less risk than is inherent in manipulation through neurostimulator implant surgery or by using drugs ${ }^{19}$.

Due to the increasing incidence of mood disorders and anxiety in the global population, researchers have sought increasingly effective, safe and noninvasive investigative and therapeutic techniques, with a lower incidence of adverse effects, for these disorders. According to the World Health Organization, depression and anxiety are among the most prevalent diseases in society, as per the tenth edition of the International Classification of Diseases (ICD-10), and are considered "common mental disorders" due to their high level of comorbidity and because they have similar therapeutic approaches. Research in the clinical field is needed in order to discover more effective and less invasive new treatments, given that not all patients respond to psychopharmacological or psychotherapeutic interventions ${ }^{20}$.

\section{NONINVASIVE TRANSCRANIAL STIMULATION FOR MAJOR DEPRESSIVE DISORDER}

Transcranial magnetic stimulation has received FDA approval for the treatment of major depressive disorder ${ }^{21}$. One notable fact is that a third of major depressive disorder patients are treatment-resistant, defined by the lack of adequate response of the symptoms after two or three antidepressant treatments ${ }^{22}$. Due to the high prevalence of resistance to treatment and failed antidepressant response, the National Institute of Mental Health developed the Sequenced Treatment Alternatives to Relieve Depression Trial, a systematic protocol for treating depression. According to the latter protocol, cumulative response and remission rates after two unsuccessful antidepressant treatments are $73 \%$ and $47 \%$ respectively ${ }^{23}$. Based on these data, 
numerous repetitive TMS (rTMS) studies have been performed on the treatment of major depressive disorder and have shown positive results, suggesting that low-frequency stimulation $(1 \mathrm{~Hz})$ of the right dorsolateral prefrontal cortex, or high frequency over the left dorsolateral prefrontal cortex, both have antidepressant effects ${ }^{24}$.

Double-blind studies have been developed using anodal tDCS on the left dorsolateral prefrontal cortex, with low intensities between 1 and $2 \mathrm{~mA}$, over 10 days or more, and have shown positive results in reducing depressive symptoms ${ }^{25,26}$. In the study by Fregni et al. ${ }^{25}$, the results indicated a $69 \%$ improvement in symptoms of depression after just five sessions of tDCS, after 1.5 weeks, compared to a $30 \%$ improvement in the control group, measured on the Hamilton Depression Rating Scale. The same results were found by Boggio et al. ${ }^{26}$, with an improvement of $40.5 \%$ in the group that received tDCS, in comparison to $10.4 \%$ of the control group, after two weeks of treatment. Taking into account these promising results of tDCS as an antidepressant treatment, replication of these studies in the future is suggested.

\section{EFFECTS OF TRANSCRANIAL STIMULATION UPON ANXIETY DISORDERS}

In addition to depression, the effects of transcranial stimulation on anxiety disorders and related disorders have also been investigated. However, before discussing some of these studies, it should be noted that, based on the fifth edition of the Diagnostic and Statistical Manual of Mental Disorders (DSM-V, 2014), published by the American Psychiatric Association, some disorders such as anxiety disorders, classically recognized by the international community, have been registered separately in this latest edition of the DSM, in their own chapters, with separate nosologies. Among these are obsessive-compulsive disorder (OCD), post-traumatic stress disorders (PTSD) and acute stress. This new and controversial classification has been the target of criticism, but it is not the purpose of this review to analyze its pros and cons. Thus, for the description and analysis of some of the main studies investigating the effects of transcranial stimulation on OCD and PTSD, before and after the publication of the DSM-V, a purely descriptive analysis of their clinical results is adopted, without considering whether or not they are anxiety disorders according to this version of the DSM.

\section{NEUROMODULATION AND OBSESSIVE-COMPULSIVE DISORDER}

Obsessive-compulsive disorder is characterized by the presence of obsessions and/or compulsions, where obsessions are thoughts, impulses or recurrent and persistent images that are intrusive and unwanted experiences, while compulsions are repetitive behaviors or mental acts that an individual feels compelled to perform in response to an obsession or according to a set of rules that must be rigidly applied. Because it is a chronic disease characterized by obsessions and compulsions, OCD causes discomfort to the patient and his/her family ${ }^{27}$ and was ranked as the fourth most common psychiatric disorder, affecting approximately $1-3 \%$ of the global population ${ }^{28}$. It is also known that the disease is associated with dysfunction in the frontostriatal circuit, including the dorsolateral prefrontal cortex, orbitofrontal cortex, and medial prefrontal cortex, as well as the supplementary motor area, supplemental gyrus, anterior cingulate gyrus and basal ganglia ${ }^{29}$. An interesting fact is that only $40-60 \%$ of patients respond to pharmacological treatment and cognitive behavioral therapy ${ }^{30}$, justifying the use of new techniques in order to reduce resistance to treatment in these patients. Further research is still needed to provide a better understanding of the neural circuits involved in this disorder, since aspects of its etiology and pathophysiology remain unknown. Recent studies with rTMS emphasize that inhibitory application at a low frequency $(1 \mathrm{~Hz})$ on the supplementary motor area improves symptoms and increases the motor threshold ${ }^{31}$ and intracortical inhibition measured by the matched magnetic pulses technique ${ }^{32}$, the latter considered to be dependent on GABAergic mechanisms. These results corroborate the findings of Gomes et al. ${ }^{33}$ who, in a recent randomized, double-blind study of OCD patients, showed that those who received rTMS on the supplementary motor area over two weeks had $35 \%$ improvement in OCD symptoms (Y-BOCS scale). Also in relation to symptoms of anxiety, there was a reduction in symptoms of $19.6 \%$ in the tDCS group compared to $9.5 \%$ in the group undergoing control stimulation, according to the anxiety scale (Hamilton Rating Scale for Anxiety - HRAS-14) after two weeks of stimulation. As to the symptoms of depression, they found no significant difference between tDCS and control groups on the Hamilton Scale for Depression (HRSD-24). In recent literature reviews, Berlim et al. ${ }^{34}$ and Senço et al. ${ }^{35}$ also observed that response rates among patients who received low-frequency rTMS on the orbitofrontal cortex or supplementary motor area vary between $35 \%$ and $13 \%$, making these areas promising targets for reducing the symptoms of OCD, but studies are still incipient, as pointed out by some authors ${ }^{14}$.

\section{NEUROMODULATION AND POST-TRAUMATIC STRESS DISORDER}

In addition to OCD, a few studies have also investigated the effects of transcranial stimulation on PTSD. This is a psychiatric condition that occurs in people who have 
witnessed events involving active threat of death or serious injury, or a threat to the physical safety of themselves or others ${ }^{36}$ and is characterized by three symptom clusters: re-experience, avoidance and hyperarousal, resulting in social or occupational dysfunction. The symptoms must be present for at least a month, and may last for years. It is also a condition in which exposure to a risk against life results in a set of intrusive memories, where the individual experiences events associated with stress ${ }^{37}$. Epidemiological studies estimate that $7.8 \%$ of the US population suffer from PTSD at some point in their lives ${ }^{38}$, suggesting a psychosocial and economic loss exceeding US\$ 3 billion in lost productivity to the US every year ${ }^{36}$.

Pharmacological studies have shown that reduced glutamatergic neurotransmission, by AMPA receptor blockage, results in anxiolytic effects. In PTSD patients, this effect results in reduced flashbacks and nightmares, typical symptoms of the disorder. Regarding the hypothalamicpituitary-adrenal axis, hypocortisolemia is different from other anxiety disorders. It is not exactly known why the reduction occurs, but it has been found that when cortisol levels are lower in PTSD, the severity of symptoms is greater. However, although many drugs have demonstrated therapeutic benefit in humans with PTSD $^{39}$ and many of these drugs have been shown to be capable of preventing anxious behavior and cognitive impairment in rats with stress ${ }^{40}$, not all patients respond to pharmacological treatment options for PTSD. For this reason, non-pharmacological treatments and noninvasive methods such as rTMS and tDCS have been tried ${ }^{41}$.

A few studies have shown that administration of low-frequency rTMS on the right dorsolateral prefrontal cortex is beneficial for improving the symptoms of $\mathrm{PTSD}^{9}$, by decreasing cortical excitability. Garcia-Toro et al. ${ }^{42}$ demonstrated that refractory PTSD patients showed an improvement of clinical symptoms after 10 sessions of low-frequency rTMS $(1 \mathrm{~Hz})$ applied to the right dorsolateral prefrontal cortex. In a complementary manner, Osuch et al. ${ }^{43}$ demonstrated that patients who had 20 sessions of low-frequency rTMS $(1 \mathrm{~Hz})$ on the right dorsolateral prefrontal cortex and PTSD exposure therapy showed a reduction in the symptomatic and psychophysiological effects of PTSD, as assessed by the Hamilton Depression Rating Scale, the Impact of Events Scale and the Clinical Administered PTSD Scale. Curiously, a group of researchers has been conducting experiments using high-frequency rTMS $(10 \mathrm{~Hz})$ instead of low frequency rTMS in patients with PTSD, and has also reported positive results ${ }^{9}$. Such experiments, however, still need to be replicated by other researchers, on the basis of variable conflicting results.

\section{Correlation with neuroimaging studies}

Neuroimaging studies on rTMS and PTSD have shown increased oxygenation in the right prefrontal cortex when participants are exposed to experiences that remind them of the traumatic experiences, suggesting that overactivity on the right side in PTSD is associated with the role of the right hemisphere in anxiety and other adverse emotional experiences $^{44}$. Thus, low-frequency rTMS (1 Hz) might decrease activity in the cortical areas of the right hemisphere, which in turn might improve the abnormalities and reduce cerebral functional asymmetries associated with PTSD ${ }^{43}$. Still, hyperactivation of the amygdala and the rostral region of the cingulate cortex is observed in these patients, and the higher the activation of these regions, the greater the severity of the symptoms of PTSD.

These findings therefore suggest that rTMS on the right dorsolateral prefrontal cortex seems to be well suited to become an effective tool in the treatment of PTSD; moreover, there is evidence that rTMS produces anxiolytic action in humans ${ }^{45}$. Berlim et al. ${ }^{46}$ reviewed several randomized controlled studies conducted between 1995 and 2013 and found that studies on PTSD patients in which rTMS was applied on the right dorsolateral prefrontal cortex pointed to significant differences from patients undergoing sham rTMS. In addition, there were differences in the symptoms of anxiety and depression among PTSD patients before and after rTMS. Additionally, Karsen et al. ${ }^{47}$ conducted a review of published reports including a total of 132 patients to evaluate the efficacy of rTMS in the treatment of PTSD. Based on the review, it was found that the variables most often studied were: a) treatment of the right or left cerebral hemisphere; b) stimulation frequency $(0.3,1,5,10$ or $20 \mathrm{~Hz})$; c) anatomical location; d) number of stimulation pulses; e) combination of rTMS with exposure. All studies stimulated pre-frontal regions.

\section{Neuromodulation for PTSD and the right-left dilemma}

Boggio et al. ${ }^{48}$ demonstrated that high-frequency rTMS $(20 \mathrm{~Hz})$ of either the right or left cerebral hemisphere can be effective in reducing the symptoms of PTSD. In contrast, some authors have observed that low-frequency rTMS $(1 \mathrm{~Hz})$ on the right dorsolateral prefrontal cortex ${ }^{43}$ seems to be more effective. Again, however, Cohen et al. ${ }^{9}$, upon comparing low-frequency rTMS (1Hz) and high-frequency rTMS $(10 \mathrm{~Hz})$ on the right dorsolateral prefrontal cortex, concluded that high-frequency rTMS showed greater improvement of PTSD symptoms compared to low-frequency rTMS (29.3\% and 10.4\% respectively). Furthermore, Rosenberg et al. ${ }^{49}$ compared the effect of low-frequency and high-frequency rTMS (1 Hz and $5 \mathrm{~Hz}$, respectively) and found low-frequency rTMS on the left dorsolateral prefrontal cortex to be efficient in reducing depressive symptoms, but not PTSD symptoms.

Although studies have demonstrated effects on cortical activation for both high-frequency and low-frequency stimulation, and treatment of both the right and left dorsolateral prefrontal cortices have been shown to reduce 
symptoms of PTSD, it is not yet understood how stimulation of the dorsolateral prefrontal cortex affects this neural circuitry. Regarding the rTMS mechanism of action in PTSD, it is known that: a) stimulating the right dorsolateral prefrontal cortex with a high frequency activates the hypothalamic-pituitary-adrenal axis, inhibiting excessive autonomic response and suppressing activity of the amygdala ${ }^{9}$; b) stimulating the right dorsolateral prefrontal cortex with a low frequency inhibits the right hemisphere, reducing hyperactivity of the right prefrontal cortex in patients with PTSD. Thus, both low-frequency and high-frequency rTMS, when applied to the right side, are potentially well suited to reduce the symptoms of PTSD.

\section{Limitations of PTSD and PTSD-depression studies}

Regarding the limitations of PTSD studies, Karsen et al. ${ }^{47}$ suggest that, although there are studies in which both frequencies can contribute to a decrease in the symptoms of PTSD, one frequency may be better than the other, and further research is therefore needed in this area. Furthermore, the small number of subjects in these analyses limits any generalization of the findings, which increases the need for further studies with larger samples and improved stimulation parameters $^{50}$; moreover, the mechanism of action of rTMS in anxiety disorders needs to be further clarified, by combining TMS with neuroimaging ${ }^{51}$.

In addition to treatment with rTMS, PTSD is also treated through exposure to objects and events that induce anxiety and memories of aversive episodes ${ }^{52}$, but in a controlled environment so that patients know that they are not in danger. Finally, the frequent coexistence of anxiety and mood disorders justifies the concomitant study, for example, of panic disorder (PD) and major depressive disorders with rTMS. Many researchers have been interested in both psychiatric disorders, since they lead to a decrease in functionality, resulting in increased morbidity and suicide rates ${ }^{53}$.

\section{NEUROMODULATION AND PANIC DISORDER}

Although less numerous, studies have also been published on the effects of transcranial stimulation in patients with PD. For example, low-frequency rTMS $(1 \mathrm{~Hz})$ on the right dorsolateral prefrontal cortex seems to lessen the symptoms of panic in these patients ${ }^{32}$. There seems to be an activation asymmetry, so that, in PD patients, the right hemisphere appears to be more active than the left in the dorsolateral prefrontal cortex area ${ }^{54}$.

\section{Right-sided low-frequency rTMS and PD}

To test the clinical effects of low-frequency rTMS $(1 \mathrm{~Hz})$ on the right dorsolateral prefrontal cortex of patients with PD and depression who are resistant or intolerant to medication, Zwanzger et al. ${ }^{55}$ observed that after two weeks of rTMS treatment there was significant improvement in symptoms of anxiety and depression, corroborating the findings of Mantovani et al. ${ }^{56}$ Later, Mantovani et al. ${ }^{32}$ demonstrated that, after four weeks of low-frequency rTMS stimulation of the right dorsolateral prefrontal cortex, patients who received real treatment fared better than those who received only sham stimulation. It can therefore be concluded that low-frequency rTMS on the right dorsolateral prefrontal cortex improves symptoms of major depression and anxiety disorders ${ }^{55}$. Low-frequency rTMS $(1 \mathrm{~Hz})$ on the right dorsolateral prefrontal cortex decreased symptoms of panic according to Li et al..$^{57}$, although the difference was not significant between patients who received rTMS and sham rTMS, measured by the Panic Disorder Severity Scale.

\section{Left-sided high-frequency rTMS stimulation and PD}

In contrast, Pallanti and Bernardi ${ }^{50}$ concluded that high-frequency rTMS on the right dorsolateral prefrontal cortex reduced symptoms in anxiety disorders and had positive results in patients with PTSD and PD, while the case study by Sakkas et al. ${ }^{58}$ in which high-frequency rTMS (10-20Hz) was administered to the left dorsolateral prefrontal cortex in a patients with PD who was resistant to pharmacological treatment, also showed an improvement in symptoms. These findings are in keeping with other studies that compared high-frequency rTMS over the left dorsolateral prefrontal cortex to the effects of antidepressants. Additionally, Dresler et al. ${ }^{59}$ demonstrated improvements in symptoms of patients diagnosed with PD as a result of three weeks of high-frequency rTMS over the left dorsolateral prefrontal cortex, confirming the study by Guaiana et al. ${ }^{60}$, which found an improvement in the symptoms of anxiety on the Panic Disorder Severity Scale.

A limitation of the rTMS method in anxiety disorders is that its impact is on the cortical surface layers, and it is not possible to directly stimulate more distant cortical and subcortical areas that are relevant to the pathogenesis of anxiety disorders. Thus, further studies are recommended to determine the role of tDCS in the treatment of anxiety disorders, since it is a less focal stimulation and may influence deeper neuronal circuits.

In conclusion, both TMS and tDCS appear to be safe and useful neuromodulatory techniques with potential application in the treatment of anxiety disorders, as well as a number of other neuropsychiatric disorders. However, as is well illustrated by this review of anxiety disorders, larger clinical trials are needed if consensus is to be reached regarding indications, optimal treatment protocols and clinical relevance of these non-pharmacological interventions. Morevover, caution should be exercised to avoid abusive use of these powerful neuromodulatory techniques, due to the uncertainties about their exact mechanism of action and possible long-term side effects. 
1. Barker AT, Jalinous R, Freeston IL. Non-invasive magnetic stimulation of human motor cortex. Lancet. 1985;1(8437):1106-7. doi:10.1016/S0140-6736(85)92413-4

2. Brasil-Neto JP, McShane LM, Fuhr P, Hallett M, Cohen LG. Topographic mapping of the human motor cortex with magnetic stimulation: factors affecting accuracy and reproducibility. Electroencephalogr Clin Neurophysiol. 1992;85(1):9-16. doi:10.1016/0168-5597(92)90095-S

3. Hallett M. Transcranial magnetic stimulation and the human brain. Nature. 2000;406(6792):147-50. doi:10.1038/35018000

4. Pascual-Leone A, Valls-Solé J, Brasil-Neto JP, Cohen LG, Hallett M. Akinesia in Parkinson's disease. I. Shortening of simple reaction time with focal, single-pulse transcranial magnetic stimulation. Neurology. 1994;44(5):884-91. doi:10.1212/WNL.44.5.884

5. Fregni F, Otachi PTM, Do Valle A, Boggio PS, Thut G, Rigonatti SP et al. A randomized clinical trial of repetitive transcranial magnetic stimulation in patients with refractory epilepsy. Ann Neurol. 2006;60(4):447-55. doi:10.1002/ana.20950

6. Pascual-Leone A, Rubio B, Pallardó F, Catalá MD. Rapid-rate transcranial magnetic stimulation of left dorsolateral prefrontal cortex in drug-resistant depression. Lancet. 1996;348(9022):233-7. doi:10.1016/S0140-6736(96)01219-6

7. Grisaru N, Chudakov B, Yaroslavsky Y, Belmaker RH. Transcranial magnetic stimulation in mania: a controlled study. Am J Psychiatry. 1998;155(11):1608-10. doi:10.1176/ajp.155.11.1608

8. Greenberg BD, George MS, Martin JD, Benjamin J, Schlaepfer TE, Altemus $\mathrm{M}$ et al. Effect of prefrontal repetitive transcranial magnetic stimulation in obsessive-compulsive disorder: a preliminary study. Am J Psychiatry. 1997;154(6):867-9. doi:10.1176/ajp.154.6.867

9. Cohen H, Kaplan Z, Kotler M, Kouperman I, Moisa R, Grisaru N. Repetitive transcranial magnetic stimulation of the right dorsolateral prefrontal cortex in posttraumatic stress disorder: a double-blind placebo-controlled study. Am J Psychiatry. 2004;161(3):515-24. doi:10.1176/appi.ajp.161.3.515

10. Priori A. Brain polarization in humans: a reappraisal of an old tool for prolonged non-invasive modulation of brain excitability. Clin Neurophysiol. 2003;114:589-95. doi:10.1016/S1388-2457(02)00437-6

11. Nitsche MA, Paulus W. Excitability changes induced in the human motor cortex by weak transcranial direct current stimulation. J Physiol. 2000;527(3):633-9. doi:10.1111/j.1469-7793.2000.t01-1-00633.x

12. Datta A, Bansal V, Diaz J, Patel J, Reato D, Bikson M. Gyri-precise head model of transcranial direct current stimulation: improved spatial focality using a ring electrode versus conventional rectangular pad. Brain Stimulat. 2009;2(4):201-7. doi:10.1016/j.brs.2009.03.005

13. Nitsche MA, Cohen LG, Wassermann EM, Priori A, Lang N, Antal A et al. Transcranial direct current stimulation: state of the art 2008. Brain Stimulat. 2008;1(3):206-23. doi:10.1016/j.brs.2008.06.004

14. Utz KS, Dimova V, Oppenländer K, Kerkhoff G. Electrified minds: transcranial direct current stimulation (tDCS) and galvanic vestibular stimulation (GVS) as methods of non-invasive brain stimulation in neuropsychology: a review of current data and future implications. Neuropsychologia. 2010;48(10):2789-810. doi:10.1016/j.neuropsychologia.2010.06.002

15. Hummel F, Celnik P, Giraux P, Floel A, Wu WH, Gerloff C et al. Effects of non-invasive cortical stimulation on skilled motor function in chronic stroke. Brain. 2005;128(3):490-9. doi:10.1093/brain/awh369

16. Volpato C, Piccione F, Cavinato M, Duzzi D, Schiff S, Foscolo $L$ et al. Modulation of affective symptoms and resting state activity by brain stimulation in a treatment-resistant case of obsessive-compulsive disorder. Neurocase. 2013;19(4):360-70. doi:10.1080/13554794.2012.667131
17. Nitsche MA, Liebetanz D, Lang N, Antal A, Tergau F, Paulus W. Safety criteria for transcranial direct current stimulation (tDCS) in humans. Clin Neurophysiol. 2003;114(11):2220-2. doi:10.1016/S1388-2457(03)00235-9

18. Palm U, Keeser D, Schiller C, Fintescu Z, Nitsche M, Reisinger E et al. Skin lesions after treatment with transcranial direct current stimulation (tDCS). Brain Stimulat. 2008;1(4):386-7. doi: 10.1016/j.brs.2008.04.003

19. Heinrichs $\mathrm{JH}$. The promises and perils of non-invasive brain stimulation. Int J Law Psychiatry. 2012;35(2):121-9. doi:10.1016/j.ijlp.2011.12.006

20. Vennewald N, Diemer J, Zwanzger P. Repetitive transcranial magnetic stimulation (rTMS) for anxiety disorders: a possible therapeutic option? Fortschr Neurol Psychiatr. 2013;81(10):550-60. doi:10.1055/s-0033-1335979

21. Horvath JC, Mathews J, Demitrack MA, Pascual-Leone A. The NeuroStar TMS device: conducting the FDA approved protocol for treatment of depression. J Vis Exp. 2010;(45):2345. doi:10.3791/2345

22. Nemeroff CB. Prevalence and management of treatment-resistant depression. J Clin Psychiatry. 2007;68 Suppl 8:17-25.

23. Rush AJ, Trivedi MH, Wisniewski SR, Nierenberg AA, Stewart JW, Warden D et al. Acute and longer-term outcomes in depressed outpatients requiring one or several treatment steps: a STAR*D report. Am J Psychiatry. 2006;163(11):1905-17. doi:10.1176/ajp.2006.163.11.1905

24. Brasil-Neto JP, Boechat-Barros R, Mota-Silveira DA. [The use of slow-frequency transcranial magnetic stimulation in the treatment of depression at Brasília University Hospital: preliminary findings]. Arq Neuropsiquiatr. 2003;61(1):83-6. Portuguese. doi:10.1590/S0004-282X2003000100015

25. Fregni F, Boggio PS, Nitsche MA, Marcolin MA, Rigonatti SP, Pascual-Leone A. Treatment of major depression with transcranial direct current stimulation. Bipolar Disord. 2006;8(2):203-4. doi:10.1111/j.1399-5618.2006.00291.x

26. Boggio PS, Rigonatti SP, Ribeiro RB, Myczkowski ML, Nitsche MA, Pascual-Leone A et al. A randomized, double-blind clinical trial on the efficacy of cortical direct current stimulation for the treatment of major depression. Int J Neuropsychopharmacol. 2008;11(2):249-54. doi:10.1017/S1461145707007833

27. Vikas A, Avasthi A, Sharan P. Psychosocial impact of obsessive-compulsive disorder on patients and their caregivers: a comparative study with depressive disorder. Int J Soc Psychiatry. 2011;57(1):45-56. doi:10.1177/0020764009347333

28. Abramowitz JS, Taylor S, McKay D. Obsessive-compulsive disorder. Lancet. 2009;374(9688):491-9. doi:10.1016/S0140-6736(09)60240-3

29. Yücel M, Harrison BJ, Wood SJ, Fornito A, Wellard RM, Pujol $J$ et al. Functional and biochemical alterations of the medial frontal cortex in obsessive-compulsive disorder. Arch Gen Psychiatry. 2007;64(8):946-55. doi:10.1001/archpsyc.64.8.946

30. Pallanti S, Hollander E, Bienstock C, Koran L, Leckman J, Marazziti D et al. International Treatment Refractory OCD Consortium: Treatment non-response in OCD: methodological issues and operational definitions. Int J Neuropsychopharmacol. 2002;5(2):181-91. doi:10.1017/S1461145702002900

31. Mantovani A, Simpson HB, Fallon BA, Rossi S, Lisanby SH. Randomized sham-controlled trial of repetitive transcranial magnetic stimulation in treatment-resistant obsessive-compulsive disorder. Int J Neuropsychopharmacol. 2010;13(2):217-27. doi:10.1017/S1461145709990435

32. Mantovani A, Aly M, Dagan Y, Allart A, Lisanby SH. Randomized sham controlled trial of repetitive transcranial magnetic stimulation to the dorsolateral prefrontal cortex for the treatment of panic disorder with comorbid major depression. J Affect Disord. 2013;144(1-2):153-9. doi:10.1016/j.jad.2012.05.038 
33. Gomes PVO, Brasil-Neto JP, Allam N, Souza ER. A randomized, double-blind trial of repetitive transcranial magnetic stimulation in obsessive-compulsive disorder with three-month follow-up. J Neuropsychiatry Clin Neurosci. 2012;24(4):437-43. doi:10.1176/appi.neuropsych.11100242

34. Berlim MT, Neufeld NH, Van den Eynde F. Repetitive transcranial magnetic stimulation (rTMS) for obsessive-compulsive disorder (OCD): an exploratory meta-analysis of randomized and sham-controlled trials. J Psychiatr Res. 2013;47(8):999-1006. doi:10.1016/j.jpsychires.2013.03.022

35. Senço NM, Huang Y, D’Urso G, Parra LC, Bikson M, Mantovani A et al. Transcranial direct current stimulation in obsessive-compulsive disorder: emerging clinical evidence and considerations for optimal montage of electrodes. Exp Rev Med Devices Early online. 2015;12(4):381-91. doi:10.1586/17434440.2015.1037832

36. American Psychiatric Association. Diagnostic and statistical manual of mental disorders DSM-5. 5th ed. Washington, DC: American Psychiatry Association; 2014.

37. Nemeroff CB, Bremner JD, Foa EB, Mayberg HS, North CS Posttraumatic stress disorder: a state-of-the-science review. J Psychiatry Res. 2006;40(1):1-21. doi:10.1016/j.jpsychires.2005.07.005

38. Kessler RC, Sonnega A, Bromet E, Hughes M, Nelson CB. Posttraumatic stress disorder in the National Comorbidity Survey. Arch Gen Psychiatry. 1995;52(12):1048-60. doi:10.1001/archpsyc.1995.03950240066012

39. Tucker P, Zaninelli R, Yehuda R, Ruggiero L, Dillingham K, Pitts CD. Paroxetine in the treatment of chronic posttraumatic stress disorder: results of a placebo-controlled, flexible-dosage trial.J Clin Psychiatry. 2001;62(11):860-8. doi:10.4088/JCP.v62n1105

40. Wang HN, Peng Y, Tan QR, Chen YC, Zhang RG, Qiao YT et al. Quetiapine ameliorates anxiety-like behavior and cognitive impairments in stressed rats: implications for the treatment of posttraumatic stress disorder. Physiol Res. 2010;59(2):263-71. doi:10.1037/e717702011-016

41. McCann UD, Kimbrell TA, Morgan CM, Anderson T, Geraci M, Benson BE et al. Repetitive transcranial magnetic stimulation for posttraumatic stress disorder. Arch Gen Psychiatry. 1998;55(3):276-79. doi:10.1001/archpsyc.55.3.276

42. García-Toro M, Salva Coll J, Crespí Font M, Andrés Tauler J, Aguirre Orue I, Bosch Calero C. [Panic disorder and transcranial magnetic stimulation]. Actas Esp Psiquiatr. 2002;30(4):221-4. Spanish.

43. Osuch EA, Benson BE, Luckenbaugh DA, Geraci M, Post RM, McCann U. Repetitive TMS combined with exposure therapy for PTSD: a preliminary study. J Anxiety Disord. 2009;23(1):54-9. doi:10.1016/j.janxdis.2008.03.015

44. Rauch SL, van der Kolk BA, Fisler RE, Alpert NM, Orr SP, Savage $\mathrm{CR}$ et al. A symptom provocation study of posttraumatic stress disorder using positron emission tomography and script-driven imagery. Arch Gen Psychiatry. 1996;53(5):380-7. doi:10.1001/archpsyc.1996.01830050014003

45. Zwanzger P, Fallgatter AJ, Zavorotnyy M, Padberg F. Anxiolytic effects of transcranial magnetic stimulation: an alternative treatment option in anxiety disorders? J Neural Transm (Vienna). 2009;116(6):767-75. doi:10.1007/s00702-008-0162-0

46. Berlim MT, Van den Eynde F, Daskalakis ZJ. Clinically meaningful efficacy and acceptability of low-frequency repetitive transcranial magnetic stimulation (rTMS) for treating primary major depression: a meta-analysis of randomized, double-blind and sham-controlled trials. Neuropsychopharmacology. 2013;38(4):543-51. doi:10.1038/npp.2012.237
47. Karsen EF, Watts BV, Holtzheimer PE. Review of the effectiveness of transcranial magnetic stimulation for pos-traumatic stress disorder. Brain Stimul. 2014;4(2):151-7. doi:10.1016/j.brs.2013.10.006

48. Boggio PS, Rocha M, Oliveira MO, Fecteau S, Cohen RB, Campanhã $\mathrm{C}$ et al. Noninvasive brain stimulation with high-frequency and low-intensity repetitive transcranial magnetic stimulation treatment for posttraumatic stress disorder. J Clin Psychiatry. 2010;71(8):992-9. doi:10.4088/JCP.08m04638blu

49. Rosenberg PB, Mehndiratta RB, Mehndiratta YP, Wamer A, Rosse RB, Balish M. Repetitive transcranial magnetic stimulation treatment of comorbid posttraumatic stress disorder and major depression. J Neuropsychiatry Clin Neurosci. 2002;14(3):270-6. doi:10.1176/jnp.14.3.270

50. Pallanti S, Bernardi S. Neurobiology of repeated transcranial magnetic stimulation in the treatment of anxiety: a critical review. Int Clin Psychopharmacol. 2009;24(4):163-73. doi:10.1097/YIC.0b013e32832c2639

51. Paes F, Machado S, Arias-Carrión O, Velasques B, Teixeira S, Budde $\mathrm{H}$ et al. The value of repetitive transcranial magnetic stimulation (rTMS) for the treatment of anxiety disorders: an integrative review. CNS Neurol Disord Drug Targets. 2011;10(5):610-20. doi:10.2174/187152711796234943

52. Echeburúa E, de Corral P, Zubizarreta I, Sarasua B. Psychological treatment of chronic posttraumatic stress disorder in victims of sexual aggression. Behav Modif. 1997;21(4):433-56. doi:10.1177/01454455970214003

53. Katz C, Yaseen ZS, Mojtabai R, Cohen LJ, Galynker II. Panic as an independent risk factor for suicide attempt in depressive illness: findings from the National Epidemiological Survey on Alcohol and Related Conditions (NESARC). J Clin Psychiatry. 2011;72(12):1628-35. doi:10.4088/JCP.10m06186blu

54. Heuvel OA, Veltman DJ, Groenewegen HJ, Witter MP, Merkelbach $J$, Cath DC et al. Disorder-specific neuroanatomical correlates of attentional bias in obsessive-compulsive disorder, panic disorder, and hypochondriasis. Arch Gen Psychiatry. 2005;62(8):922-33. doi:10.1001/archpsyc.62.8.922

55. Zwanzger P, Minov C, Ella R, Schüle C, Baghai T, Möller HJ et al. Transcranial magnetic stimulation for panic. Am J Psychiatry. 2002;159(2):315-6. doi:10.1176/appi.ajp.159.2.315-a

56. Mantovani A, S.H. L, Pieraccini F, Castrogiovanni P, Rossi S. Repetitive Transcranial Magnetic Stimulation (rTMS) in the treatment of panic disorder (PD) with comorbid major depression. J Affect Disord. 2007;102(1-3):277-80. doi:10.1016/j.jad.2006.11.027

57. Li H, Wang J, Li C, Xiao Z. Repetitive transcranial magnetic stimulation (rTMS) for panic disorder in adults. Cochrane Database Syst Rev. 2014;(9):c009083. doi:10.1002/14651858.CD009083.pub2

58. Sakkas P, Psarros C, Papadimitriou GN, Theleritis CG, Soldatos CR. Repetitive transcranial magnetic stimulation (rTMS) in a patient suffering from comorbid depression and panic disorder following a myocardial infarction. Prog Neuropsychopharmacol Biol Psychiatry. 2006;30(5):960-2. doi:10.1016/j.pnpbp.2006.01.023

59. Dresler T, Ehlis AC, Plichta MM, Richter MM, Jabs B, Lesch KP et al. Panic disorder and a possible treatment approach by means of high-frequency rTMS: a case report. World J Biol Psychiatry. 2009;10(4-3):991-7. doi:10.1080/15622970902898147

60. Guaiana G, Mortimer AM, Robertson C. Efficacy of transcranial magnetic stimulation in panic disorder: a case report. Aust N Z J Psychiatry. 2005;39(11-12):1047. doi:10.1080/j.1440-1614.2005.01724_3.x 